\title{
Evaluación de materiales promisorios de plátano y banano en el municipio de Bituima (Cundinamarca)
}

\section{Evaluation of promising materials of plantain and banana in the municipality of Bituima (Cundinamarca)}

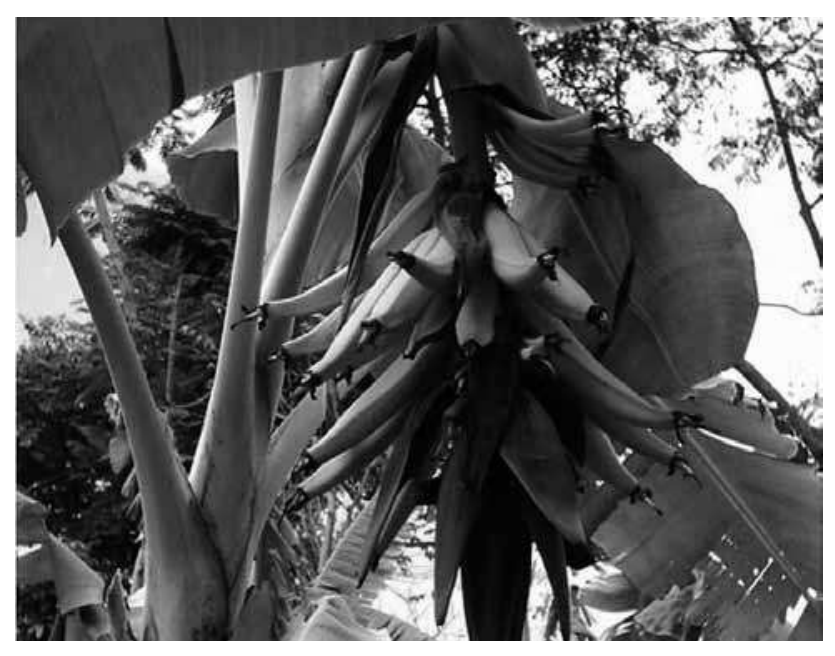

\section{RESUMEN}

En los últimos años, la Fundación Hondureña de Investigación Agrícola (FHIA) ha puesto a disposición de los países productores de banano y plátano, varios cultivares híbridos con alto potencial productivo, resistentes a Sigatoka negra y a otras plagas importantes. A partir de esto se evaluó el desempeño fisiológico y productivo de siete materiales promisorios con el fin de ofrecer alternativas nuevas para mejorar la producción de plátano y banano en el municipio de Bituima (Cundinamarca). Se evaluaron cuatro híbridos (FHIA 3, FHIA 4, FHIA 20, FHIA 21), una variedad (África 1) y las variedades locales banano y plátano como testigos, con tres repeticiones cada una, bajo un diseño de bloques completamente al azar. Se presentaron diferencias estadísticas significativas en el perímetro del seudotallo entre los híbridos (FHIA 4, FHIA 20, FHIA 21), la variedad África 1 y los testigos (plátano Dominico-Hartón y banano); en el peso del racimo entre la variedad África 1 y el banano. El híbrido FHIA 3 presentó la mayor tasa de emisión foliar (0,13 hojas/día) y el híbrido FHIA 21 la menor (0,09 hojas/día). Los híbridos tuvieron tasas similares de crecimiento que las variedades locales de banano (Gros Michel) y plátano (Dominico-Hartón), por lo cual, pueden ser adoptadas por los agricultores debido a que estas introducciones mostraron un buen desempeño fisiológico y productivo en los sistemas de producción de la zona estudiada.

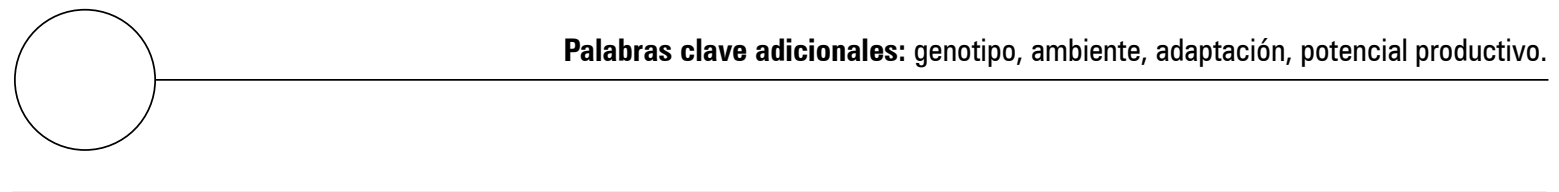

Facultad de Agronomía, Departamento de Agronomía, Universidad Nacional de Colombia, Bogotá.

2 Autor para correspondencia. cecarranzag@unal.edu.co 


\section{ABSTRACT}

In the last few years, the Honduran Foundation for Agriculture Research (FHIA) has put on the market some hybrid cultivars for banana and plantain producing countries, these new cultivars have a high production potential and are resistant to black Sigatoka and other important pests. For these reasons, the present study evaluated the physiological and productive performance of seven promising materials, in order to offer some new alternatives to improve plantain and banana production in the village of Bituima (Cundinamarca). Four hybrids and one variety were evaluated, with local banana and plantain varieties as controls, with three repetitions using a random block design. There were significant differences in the pseudostem between the hybrids FHIA 4, FHIA 20, FHIA 21, the variety Africa 1 and the controls (plantain Dominico-Harton and banana); and in bunch weight between the variety Africa 1 and the banana. The hybrid FHIA 3 presented the largest foliar emission rate (0.13 leaves per day) and the hybrid FHIA 21 the lowest (0.09 leaves per day). The hybrids had similar growth rates as the local varieties of banana (Gros Michel) and plantain (DominicoHartón), and so they can be adopted by the farmers in the studied zone to achieve good physiological and productive performance in the production systems.

Additional key words: genotype, environment, adaptation, productive potential.

Fecha de recepción: 08-02-2011

Aprobado para publicación: 27-05-2011

INTRODUCCIÓN

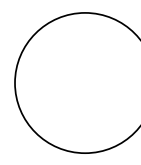

En los últimos años, la Fundación Hondureña de Investigación Agrícola (FHIA) ha puesto a disposición de los países productores de banano y plátano, varios cultivares híbridos con alto potencial productivo, resistentes a Mycosphaerella fijiensis (Sigatoka negra) y a otras plagas importantes. Estos híbridos en su mayoría tetraploides (AAAA, AAAB, AABB), son una nueva e importante alternativa para millones de personas que utilizan estos cultivos como fuente de alimento e ingresos y que actualmente están imposibilitados para enfrentar los altos costos que representa la lucha con productos químicos contra enfermedades y plagas que afectan negativamente a los clones tradicionales de banano y plátano (Álvarez et al., 2004).

El híbrido FHIA 3 (Musa cv. AABB) es un banano de cocción enano tipo Blugooe, que fue desarrollado en 1987. Tiene un alto potencial para varios países del África del Oeste y Este, donde existen muchas áreas marginales que no permiten un buen desarrollo del banano Cavendish. En la actualidad se encuentra en producción comercial en Cuba y también distribuido ampliamente en Burundi, Camerún y Nigeria entre otros países africanos (FHIA, 1993). La planta mide entre 2,5 y 3,7 m; tiene hojas decumbentes y un tallo opaco. El racimo es cilíndrico y cuelga verticalmente a la planta. Los frutos de color verde, son rectos en la parte distal y presentan ápice puntiagudo. Por ser una planta de porte bajo y muy resistente, puede sostener racimos de hasta $50 \mathrm{~kg}$ sin soporte. El híbrido FHIA 3 es resistente al marchitamiento por Fusarium y tolerante al picudo negro (Nowakunda et al., 2000).

Desde la siembra hasta la floración pueden transcurrir entre 271 y $307 \mathrm{~d}$; el primer ciclo productivo es de 100 a $110 \mathrm{~d}$. La segunda floración se presenta de los 430 a 530 d después de la siembra. El peso neto del racimo está entre 30 y $40 \mathrm{~kg}$ ( $\sin$ 
raquis), con entre 198 a 226 dedos por racimo y el peso de los dedos individuales oscila entre 155 y 179 g. Este híbrido es resistente a Fusarium oxysporum f.sp. cubense (Mal de Panamá), M. fijiensis Morelet y Ralstonia solanacearum (Moko), además presenta resistencia parcial al nematodo Radopholus similis, pero es susceptible al nematodo Pratylenchus coffeae (FHIA, 1993).

El híbrido FHIA 20 (Musa cv. AAAB) es un plátano tipo French (Dominico) que fue desarrollado en 1989. En la actualidad se encuentra en producción comercial en Honduras y otros países de Latinoamérica y El Caribe (LAC). La planta mide entre 3,2 y 4,5 m; tiene hojas decumbentes y un tallo opaco. El racimo cuelga verticalmente y tiene una forma asimétrica. Los frutos de color verde son rectos en la parte distal y el ápice del fruto tiene forma de cuello de botella. El tiempo desde la siembra hasta la floración es de 300 a 380 d, bajo condiciones de Honduras. El periodo de llenado del racimo (de parición a cosecha) es de 84 a $91 \mathrm{~d}$, el peso neto del racimo está entre 20 y $30 \mathrm{~kg}$, entre 130 y 160 dedos por racimo, y el peso de los dedos individuales entre 250 y 370 g. Este híbrido es resistente a la $M$. fijiensis Morelet y a F. oxisporum f.sp. cubense., pero susceptible a los dos nematodos más importantes: $R$. similis y $P$. coffeae (FHIA, 1993).

El híbrido FHIA 21 (Musa cv. AAAB) es un plátano tipo French (Dominico) desarrollado en 1987. En la actualidad se encuentra en producción comercial en Cuba, Honduras, Nicaragua, Guatemala, Venezuela, Ecuador, Perú, Colombia y otros países de LAC. La planta mide entre 3,5 y 4,0 m; tiene hojas decumbentes y un tallo brillante. El racimo es ligeramente inclinado y asimétrico; los frutos son de color verde claro y rectos hacia la punta, la cual presenta una forma ligeramente puntiaguda; la cáscara es más fácil de remover, pero también es susceptible a los golpes. El tiempo entre la siembra y la floración es de 240 a $280 \mathrm{~d}$ y el periodo de llenado del racimo de 85 a 100 d; el peso neto del racimo es de 22 a $27 \mathrm{~kg}$, con 120 a 150 dedos por racimo y peso individual entre 250 y 350 g. Este híbrido es resistente a la $M$. fijiensis Morelet y a F. oxysporum f.sp. cubense, sin embargo, es susceptible a los dos nematodos de mayor importancia: $R$. similis y $P$. coffeae (FHIA, 1993).

La Federación Nacional de Cafeteros introdujo un grupo de variedades no mejoradas de África Occidental (Camerún) las cuales se evaluaron en varias localidades, comparándolas con las variedades locales. Una de las más promisorias en todas las pruebas preliminares fue la variedad África 1 (Grisales, 2001), inicialmente registrada como MBOURUKOU (CIRAD, 1998). África 1 (Musa cv. AAB) es una variedad de porte medio (3,53 m), color verde del seudotallo, sin cera; longitud del falso internudo de $24 \pm 2 \mathrm{~cm}$ y ángulo de inserción foliar de $40-55^{\circ}$. El periodo de siembra a cosecha de 14,8 meses, aproximadamente. El peso del racimo de unos $14 \mathrm{~kg}$ con cinco manos y 24 dedos distribuidos en hileras doble en las dos manos superiores y simples en las demás los dedos muestran geotropismo positivo (colgantes), son de gran tamaño (240-865 g) con poca curvatura y su rendimiento (pulpa/piel = $1,75)$ es uno de los más altos entre las variedades cultivadas. Esta variedad es susceptible a $M$. musicola (Sigatoka amarilla) y $M$. fijiensis Morelet (Grisales, 2001).

Entre las variedades locales, el plátano Dominico Hartón (Musa cv. AAB), es un material bastante inestable; de acuerdo con la altitud de siembra muestra la interacción genotipo-ambiente sobre el fenotipo de la planta y su racimo. Puede alcanzar 3,3 m de altura y $18 \mathrm{~cm}$ de diámetro. Presenta un color verde claro con manchas ligeramente oscuras y los bordes de las vainas un tinte rosado suave. El número promedio es de 56 dedos. El raquis produce brácteas deciduas como todas las demás, que no subtienden ninguna flor, la bellota, aovada acuminada alargada desaparece rápidamente. El racimo es coniforme, con frutos más grandes que los del Dominico, pero más pequeños que los del Hartón. Este clon se pude cultivar sin que se afecte el tamaño del 
racimo y la calidad de la fruta, desde el nivel del mar hasta los 1.500 msnm (Belalcazar, 1991).

El clon Dominico Hartón es altamente susceptible a las sigatokas y no se tiene una variante de este plátano resistente a dichas enfermedades. Por esta razón desde hace varios años se cultivan algunos híbridos resistentes. Sin embargo no han sido evaluados sistemáticamente en cuanto al crecimiento, desarrollo y producción (ICA, 1996). Tal es el caso de los híbridos FHIA, algunos de los cuales tienen rendimientos superiores $\left(16,7 \mathrm{t} \mathrm{ha}^{-1}\right)$ en comparación con los materiales tradicionales $\left(6,7 \mathrm{t} \mathrm{ha}^{-1}\right)$ (CCI, 1999), pero que requieren de una adecuada evaluación agronómica (Belalcázar et al,. 1998). La ausencia de dichas investigaciones limita su potencial de uso como alternativa para establecer plantaciones comerciales (Herrera y Aristizábal, 2003).

El banano (Musa cv. AAA) presenta un rizoma sólido que tiene forma tuberosa o cilíndrica, con un disco caulinar muy desarrollado, corto, grueso y carnoso por la gran cantidad de parénquima amiláceo y con mucho contenido de agua (Sierra, 1993). El seudotallo aéreo se origina en el cormo y está conformado por la prolongación y modificación de las hojas, o sea por pecíolos envainadores fuertemente enrollados (Álvarez, 1981; Arbeláez, 1985). El seudotallo es de color blanco inicialmente, tornándose verde al exponerse a la luz solar. Es cilíndrico, recto y rígido (Rodríguez, et al., 1985). La planta del banano produce aproximadamente 35 hojas completas durante su crecimiento (Ovalle y Rueda, 1999). La parte basal de la lámina es obtusa y redondeada y el ápice de las hojas es truncado, los márgenes enteros y los bordes están casi paralelos en la porción media (Rodríguez et al., 1985). El fruto es una baya partenocárpica capsular indehiscente; la fruta es carnosa y suave, compuesta por tres carpelos, es de forma angulosa en estados tempranos y progresivamente cilíndrica a medida que va aumentando de grosor por la acumulación de almidón (Soto, 1985).
Este estudio tuvo como objetivo evaluar el desempeño fisiológico y productivo de siete materiales promisorios, con el fin de ofrecer nuevas alternativas para mejorar la producción de plátano y banano en el municipio de Bituima (Cundinamarca).

\section{MATERIALES Y MÉTODOS}

El experimento se ubicó en el municipio de Bituima (Cundinamarca) con una temperatura promedio de $24^{\circ} \mathrm{C}, 1.390 \mathrm{msnm}$, precipitación anual $1.500 \mathrm{~mm}$, humedad relativa del $70 \%$, a 4 $54^{\prime} 51,4^{\prime \prime} \mathrm{N}$ y 74³2'23"O. Los materiales promisorios utilizados en el experimento fueron FHIA 3, FHIA 4, FHIA 20, FHIA 21, África 1, plátano y banano como variedades locales. Se utilizó el diseño experimental de bloques completamente al azar con siete tratamientos y tres repeticiones. Las variables a evaluar fueron altura de la planta, número de hojas, perímetro del seudotallo (a $1 \mathrm{~m}$ de la base), peso del racimo, días de siembra a floración, días de floración a cosecha y días de siembra a cosecha. Los híbridos FHIA 3 y FHIA 20 fueron adquiridos de una parcela experimental del ICA en el municipio de Vianí (Cundinamarca), sembrados en plántulas de vivero. Los híbridos FHIA 4 y FHIA 21 se obtuvieron del C.I. Tulenapa de Corpoica en Carepa (Antioquia) y sembrados en sitio definitivo mediante cormos. El África 1 se adquirió del Departamento de Biología de la Universidad Nacional de Colombia, sede Bogotá en plántulas in vitro y se sembraron las plántulas endurecidas previamente. La siembra de los materiales FHIA 3, FHIA 4, FHIA 20, FHIA 21, plátano y banano se realizó en septiembre del 2004 y el África-1 en enero del 2005. Los sitios fueron estacados en triángulo a $3 \times 3 \mathrm{~m}$ teniendo 15 plantas por unidad experimental para una población de 315 plantas en total. El manejo agronómico se realizó oportunamente mediante prácticas de fertilización, deshije, desguasque, deshoje y manejo de arvenses. En la siembra, a partir de un análisis de suelos se aplicó $1,0 \mathrm{~kg}$ de cal dolomita y 2,0 kg de 
compost por sitio; al mes de sembrado se aplicó 17-6-18-2 y posteriormente, con una frecuencia de 45 días, $\mathrm{KCl}+$ urea (relación de 3:1). La comparación de los materiales con respecto a la variable número de hojas y altura de la planta se determinaron las áreas bajo la curva haciendo la integración del modelo con respecto a cada material. Con los datos generados se realizó el análisis de varianza (Anava) y una prueba de Tukey $(P \leq 0,05)$ para estimar las diferencias entre las medias. Los análisis estadísticos se realizaron con el programa estadístico "SAS" V 9.1®.

Las variables número de hojas (figura 1) y altura de planta (figura 2) de los materiales se ajustaron a un modelo de crecimiento de tipo sigmoidal de la siguiente forma:

$\mathrm{Y}=\mathrm{a} / 1+\mathrm{e}^{-\mathrm{b}(\mathrm{dds}-\mathrm{c})}$

donde:

$$
\begin{aligned}
& \mathrm{Y}=\text { número de hojas o altura } \\
& \text { a, b y c = coeficientes del modelo } \\
& \text { dds = días después de siembra }
\end{aligned}
$$

\section{RESULTADOS Y DISCUSIÓN}

En la tabla 1 se presentan los resultados del comportamiento del desarrollo de los materiales estudiados. El perímetro del seudotallo presentó diferencias altamente significativas, entre las variedades locales banano y plátano con los híbridos FHIA 21 y FHIA 4; la variedad África 1 con respecto al FHIA 4. Los días siembra a floración, floración a cosecha y siembra a cosecha no presentaron diferencias significativas, por tanto no hay relación entre periodos de crecimiento en los diferentes materiales. El peso del racimo mostró diferencias significativas entre las variedades banano Gros Michel y plátano Dominico-Hartón, y África 1 (tabla 2); en consecuencia, esta variedad local sobresalió con respecto a los híbridos, los cuales no desarrollaron su vigor híbrido en estas condiciones climáticas, por consiguiente los materiales se pueden usar en sitios donde las variedades locales estén limitadas por el ataque de patógenos.

Cabe resaltar el híbrido FHIA 3 por su precocidad con respecto a los demás materiales, lo cual sumado a su buen desempeño fisiológico, productivo y características de rusticidad y resistencia a enfermedades y plagas, se puede utilizar con éxito en un programa de diversificación para reemplazar la variedad local Cachaco que es muy susceptible a varios patógenos de importancia económica, principalmente a la enfermedad del "Moko". Similares resultados fueron encontrados por González et al. (2003), en la precocidad de FHIA 3 comparado con el Gros Michel en relación al tiempo a floración y cosecha.

Según Cottin et al. (1987), la duración de los ciclos vegetativos en banano depende del clima (principalmente la temperatura, que en el trópico está fuertemente asociada con la altitud), que produce variaciones cíclicas importantes, comprendidas entre 7 y 22 meses, y de las prácticas culturales, como riego, material vegetal, densidades y época de siembra, que también influyen en la duración del ciclo. El plátano África 1 tendió a ser más precoz en llegar a floración (430 d) y cosecha (609 d) que las otras variedades de plátano. El periodo de siembra a cosecha de la variedad África 1 (20,3 meses), bajo las condiciones de la zona experimental, difiere con otros estudios en Risaralda y Tolima donde este periodo fue de 14,8 y 16,0 meses respectivamente (Grisales, 2001); la precocidad del ciclo vegetativo de este material es una característica fundamental que le confiere tolerancia a la Sigatoka negra, pues de esta forma escapa al ataque de la enfermedad. Estudios realizados por Gómez y Castaño-Zapata (2001) en África 1 concluyeron que este material posee un alto grado de tolerancia a ambas sigatokas. Si se considera que es una variedad de reciente introducción, existe poca información publicada sobre 
su comportamiento en otras condiciones; solamente se conoce que en Urabá y el Magdalena Medio la productividad ha sido inferior a la de la zona cafetera y ha mostrado su relativa precocidad frente a las variedades tradicionales (Corpoica, 1999).
El FHIA 21 en el estudio emitió 0,63 hojas cada $8 \mathrm{~d}$ (figura 1), al momento de la floración, 2,52 $\mathrm{m}$ de altura, perímetro de seudotallo $50,4 \mathrm{~cm}$, a los 542 d salió la inflorescencia y un periodo de llenado de fruto de $152,8 \mathrm{~d}$, producción de $23 \mathrm{t}$

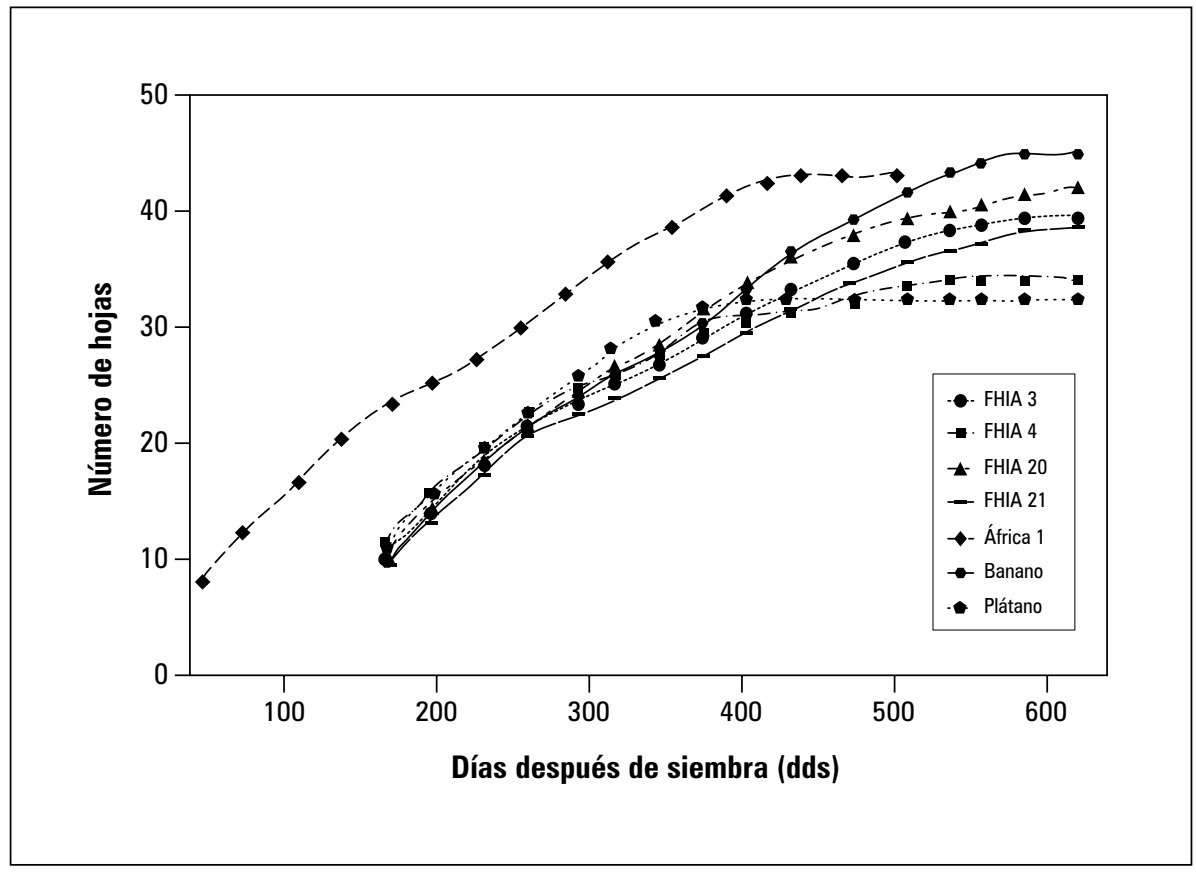

Figura 1. Comportamiento del número de hojas en siete materiales promisorios de plátano y banano en Bituima (Cundinamarca).

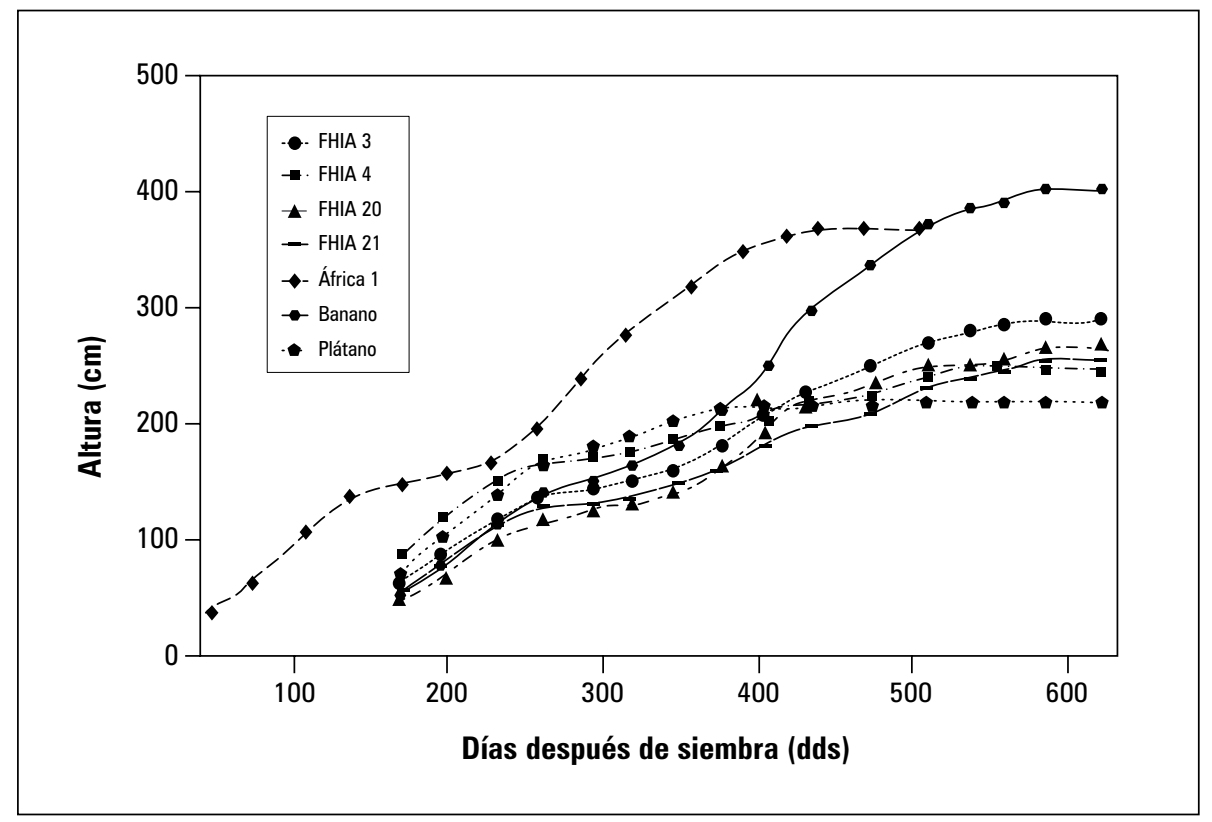

Figura 2. Comportamiento de la altura en los siete materiales promisorios en el municipio de Bituima (Cundinamarca). 
Tabla 1. Crecimiento y desarrollo de siete materiales promisorios de plátano y banano en Bituima (Cundinamarca).

\begin{tabular}{|l|c|c|c|c|c|}
\hline \multicolumn{1}{|c|}{ Tratamiento } & $\begin{array}{c}\text { Altura al momento de la } \\
\text { floración }(\mathrm{m})\end{array}$ & $\begin{array}{c}\text { Perímetro } \\
\text { seudotallo }(\mathrm{cm})\end{array}$ & $\begin{array}{c}\text { Siembra a } \\
\text { floración (días) }\end{array}$ & $\begin{array}{c}\text { Floración a } \\
\text { cosecha (días) }\end{array}$ & $\begin{array}{c}\text { Siembra a } \\
\text { cosecha } \\
\text { (días) }\end{array}$ \\
\hline FHIA 3 & $2,1 \mathrm{a}$ & $54,0 \mathrm{abc}$ & 389,5 & 159,0 & 548,5 \\
\hline FHIA 4 & $2,4 \mathrm{a}$ & $40,9 \mathrm{c}$ & 437,0 & 198,3 & 635,3 \\
\hline FHIA 20 & $2,6 \mathrm{a}$ & $59,7 \mathrm{abc}$ & 522,7 & 152,6 & 675,3 \\
\hline FHIA 21 & $2,5 \mathrm{~b}$ & $50,4 \mathrm{bc}$ & 542,0 & 152,8 & 656,8 \\
\hline África 1 & $3,6 \mathrm{a}$ & $57,5 \mathrm{~b}$ & 429,7 & 188,7 & 608,8 \\
\hline Plátano & $2,8 \mathrm{a}$ & $46,5 \mathrm{c}$ & 527,0 & 173,4 & 700,4 \\
\hline Banano & $3,9 \mathrm{a}$ & $65,8 \mathrm{a}$ & 559,3 & 120,4 & 675,8 \\
\hline CV (\%) & 11,54 & 7,44 & 12,04 & 25,46 & 7,94 \\
\hline F (tratamientos) & $*$ & $* *$ & $n .5$ & n.s \\
\hline
\end{tabular}

Promedios con letras distintas, en la misma columna, indican diferencia significativa según la prueba de Tukey $(P \leq 0,05){ }^{*},{ }^{* *}$ Prueba de $\mathrm{F}$ significativa $(P \leq 0,05, P \leq 0,01)$. n.s. Prueba de $\mathrm{F}$ no significativa. $P \leq 0,01)$. n.s. Prueba de $\mathrm{F}$ no significativa.

ha- ${ }^{-1}$. Ensayos realizados por la FHIA en condiciones de Honduras, la planta emitió una hoja cada $8 \mathrm{~d}$; al momento de la floración alcanzó una altura de $2,8 \mathrm{~m}$ y una circunferencia de seudotallo de $55 \mathrm{~cm}$, demoró $341 \mathrm{~d}$ para emitir la inflorescencia y tuvo un periodo de llenado de los frutos de 169 d y la producción del primer ciclo fue de 26,1 t ha-1 (FHIA, 1994).

No hubo diferencias significativas con respecto al número de hojas; en cuanto a la altura se presentaron diferencias significativas entre la variedad banano Gros Michel y el híbrido FHIA 21. Por tanto, el banano presenta la mayor altura (398 $\mathrm{cm})$ y el híbrido FHIA 21 la menor $(252 \mathrm{~cm})$.

En la figura 1, se aprecia que el banano Gros Michel emitió el mayor número de hojas (44) y FHIA 3 el menor de todos los materiales a los $600 \mathrm{~d}$ del ciclo. El plátano África 1 emitió todas las hojas a los $500 \mathrm{~d}$, demostrando la precocidad de su ciclo vegetativo.

La figura 2 muestra que, bajo las condiciones de la zona, el banano Gros Michel y el plátano África 1 son materiales de porte alto registrando las mayores alturas de planta, por lo tanto el manejo en fincas de ladera se torna difícil aumentando los costos de producción. Por otro lado, los híbridos (FHIA 3, FHIA 4, FHIA 21, FHIA 20) y el plátano Dominico-Hartón tienen características de porte bajo, por consiguiente estos materiales se pueden adoptar sin dificultad en los sistemas de producción de cultivos de ladera como los utilizados en el municipio de Bituima, reduciendo significativamente los costos operacionales.

Se determinó la tasa de emisión foliar (TEF) calculando la derivada del modelo anterior (figura 3). La TEF de todos los materiales se ajustó a un modelo cuadrático incrementando durante los primeros 200 a $300 \mathrm{~d}$ del ciclo y disminuyendo hacia el final del mismo. El híbrido FHIA 3 presentó la mayor TEF (0,13 hojas/d), seguido por FHIA 20, FHIA 4, Banano, Plátano, África 1 y FHIA 21. En este modelo el plátano África 1 también mostró su característica de precocidad logrando su máxima TEF y periodo vegetativo antes que los otros materiales. Bajo las condiciones agroecológicas de Bituima, el plátano Dominico-Hartón y el hibrido FHIA 21 mostraron la TEF más baja (0,09 hojas/día) aunque su periodo vegetativo fue el mismo que los otros materiales estudiados (600 d). Estu- 


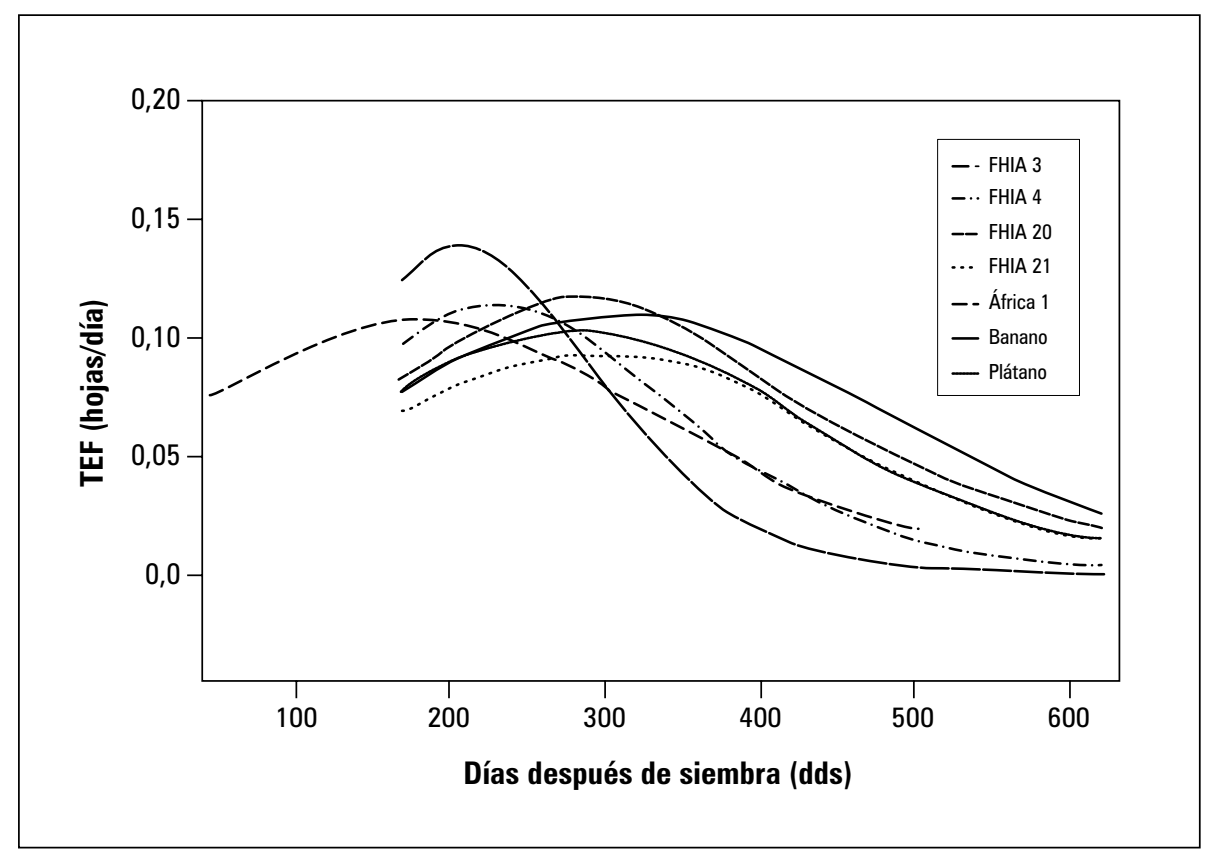

Figura 3. Comportamiento de la tasa de emisión foliar (TEF) en siete materiales promisorios de banano y plátano en Bituima (Cundinamarca).

dios realizados por Hoyos y Castaño (2007) indicaron que el comportamiento del África 1 y FHIA 3, en cuanto a la precocidad, tuvieron los menores ciclos, con valores de 324 y 392 d, respectivamente, en comparación con los materiales de referencia cuyos ciclos fueron de 384 d en Dominico-Hartón y de 439 d en Gros Michel, indicando que África 1 fue 2 meses más precoz que Dominico Hartón, y FHIA 3,47 d más precoz que Gros Michel, característica que los hace más promisorios.

En tabla 2 se observa que el peso del racimo, número de manos y dedos del banano Gros Michel fueron superiores que los demás materiales; los racimos de los híbridos (FHIA 3, FHIA 4, FHIA 20, FHIA 21) fueron similares entre sí y los de menor peso fueron los de los plátanos Dominico-Hartón y África 1. En la zona cafetera colombiana Grisales (2001) encontró que el peso del racimo de África 1 fue de $13,8 \mathrm{~kg}$, en Paraguaycito (Quindío), 14,5 kg en el Líbano (Tolima), 15,0 kg en La Catalina (Risaralda) y 12,5 kg en la Estación Central Naranjal (Caldas). En la Granja Montelindo (Caldas) el peso del racimo de África 1 fue $14,5 \mathrm{~kg}$, sin embargo esta variedad produce un racimo de mejor calidad siendo más rentable (Hoyos y Castaño, 2007). Se destacó el plátano FHIA 20 por su gran cantidad de dedos por racimo (122), mientras que el racimo de plátano África 1 es el de menor número de dedos (20). Los materiales se diferenciaron significativamente en cuanto al tamaño de los frutos, destacándose la longitud externa de los de África $1(34 \mathrm{~cm})$, pero la longitud interna de los frutos no fue diferente entre los materiales; el perímetro de los frutos es un indicador del llenado, pues este fue superior únicamente en los frutos de África 1 confirmando el mayor tamaño de los frutos de esta variedad.

El hibrido FHIA 3 presentó una mayor tasa de emisión foliar (figura 1) con respecto a los demás materiales promisorios y un porte de altura bajo (figura 2), por lo cual estos dos materiales se muestran como muy promisorios para ser adoptados 
Tabla 2. Componentes del rendimiento de siete materiales promisorios de plátano y banano en Bituima (Cundinamarca).

\begin{tabular}{|c|c|c|c|c|c|c|}
\hline \multirow{2}{*}{ Tratamiento } & \multirow{2}{*}{$\begin{array}{c}\text { Peso racimo } \\
(\mathrm{kg})\end{array}$} & \multirow{2}{*}{$\begin{array}{l}\text { Número } \\
\text { manos }\end{array}$} & \multirow{2}{*}{$\begin{array}{l}\text { Número } \\
\text { dedos }\end{array}$} & \multicolumn{2}{|c|}{ Longitud del dedo (cm) } & \multirow{2}{*}{$\begin{array}{l}\text { Perímetro del } \\
\text { dedo (cm) }\end{array}$} \\
\hline & & & & Externa & Interna & \\
\hline FHIA 3 & $19,3 a b$ & 6,3 & 84,8 & $18,0 \mathrm{e}$ & 14,0 & $16,6 b$ \\
\hline FHIA 4 & $23,3 \mathrm{ab}$ & 7,3 & 76,7 & 24,7 a & 20,3 & $16,5 b$ \\
\hline FHIA 20 & $21,9 a b$ & 8,3 & 122,0 & 18,7 ae & 14,8 & $13,9 \mathrm{~b}$ \\
\hline FHIA 21 & $20,9 \mathrm{ab}$ & 6,7 & 90,7 & 24,9 a & 20,7 & $15,4 \mathrm{a}$ \\
\hline África 1 & $13,8 \mathrm{bc}$ & 4,7 & 20,1 & $34,3 \mathrm{cb}$ & 26,3 & $20,3 \mathrm{a}$ \\
\hline Plátano & $15,5 \mathrm{bc}$ & 6,4 & 48,9 & $24,7 \mathrm{~cd}$ & 20,8 & $15,7 \mathrm{~b}$ \\
\hline Banano & $33,3 \mathrm{a}$ & 9,1 & 143,7 & $24,8 \mathrm{c}$ & 19,5 & $15,0 \mathrm{~b}$ \\
\hline CV (\%) & 25,61 & 10,85 & 17,6 & 5,48 & 9,17 & 6,4 \\
\hline $\mathrm{F}$ (tratamientos) & * & n.s & n.s & * & n.s & ** \\
\hline
\end{tabular}

Promedios con letras distintas, en la misma columna, indican diferencia significativa según la prueba de Tukey $(P \leq 0,05)^{*}$, ** Prueba de $\mathrm{F}$ significativa $(P \leq 0,05, P \leq 0,01)$. n.s. Prueba de $\mathrm{F}$ no significativa.

por los productores de Bituima en sus programas de diversificación en sus respectivas fincas.

\section{CONCLUSIONES}

El híbrido FHIA 3 presentó una mayor tasa de emisión foliar con respecto a los otros materiales evaluados, teniendo por ende ciclos de producción cortos en estas condiciones climáticas del municipio de Bituima.

ElFHIA21 presentó un periodolargo en estas condiciones, siendo un híbrido de tardío de rendimiento.
Los híbridos tienen similar tasa de crecimiento que las variedades locales de banano Gros Michel y plátano Dominico-Hartón, por lo tanto pueden ser adoptadas por los agricultores en los sistemas de producción.

Para una producción sostenible y con resistencia a los principales patógenos de importancia económica en el municipio de Bituima se recomienda el empleo del híbrido FHIA 3 y la variedad África 1, por tener ciclos de producción cortos en comparación con las variedades locales. 


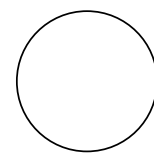

Agronet. 2010. Producción de plátano en Colombia, 1987-2009. Análisis-Estadísticas. Ministerio de Agricultura y Desarrollo Rural. En: http://www. agronet.gov.co; consulta: octubre de 2010.

Álvarez, J.M. y F.E. Rosales. 2004. Guía de campo para la identificación de los principales híbridos de banano y plátano de la FHIA. En: XVI Reunión Internacional Acorbat 2004, http://inibap.org.co, 184-185 p., consulta noviembre de 2006.

Álvarez, P.F. 1981. Cultivo de la platanera. Industria Gráfica, Madrid.

Arbeláez, D. 1985. Técnicas culturales y análisis de rentabilidad del cultivo del plátano. Conferencias. INCOLDA: Programa de Administración Agropecuaria. Manizales, Colombia.

Belalcázar, S.L., J.A. Valencia y M.I. Arcila. 1998. Conservación y evaluación de la colección colombiana de musáceas. En: Giraldo, M.J., S.L. Belalcazar y D.G. Cayón (eds). Memorias Seminario Internacional sobre Producción de Plátano. INIBAP, Corpoica, SENA, Comité de Cafeteros de Colombia, Armenia, Colombia.

Belalcázar, S.L. 1991. El cultivo del plátano en el trópico. Instituto Colombiano Agropecuario (ICA), Bogotá.

Centre Internacional en Recherche Agronomique Pour le Developpement (CIRAD), Federación Nacional de Cafeteros de Colombia, ICA, UCL. 1998. Ecofisiología de los plátanos en altitud en Colombia en relación con su susceptibilidad a las cercosporiasis. CIRAD, Montpellier, Francia.

Corpoica. 1999. Plan de investigación y transferencia para aumentar la sostenibilidad y competitividad del plátano en Colombia. Avances y resultados. Corporación Colombiana de Investigación Agropecuaria (Corpoica), Bogotá.

CCI. 1999. Boletín Informativo, julio-septiembre, 1-6. Corporación Colombia Internacional, Bogotá.

Cottin, R., P. Melin y J. Ganry. 1987. Modélisation de la production bananière. Influence de quelques paramètres en Martinique. Fruits 42(12), 691-701.

Fundación Hondureña de Investigación Agrícola. FHIA. 1994. Programa de Banano y Plátano. Informe Técnico. La Lima, Cortés, Honduras.

Fundación Hondureña de Investigación Agrícola. FHIA. 1993. Programa de banano y plátano. Informe anual. La Lima, Cortés, Honduras.

Gómez, L.A. y J. Castaño-Zapata. 2001. Manejo integrado de las Sigatoka negra (Mycosphaerella fi-
REFERENCIAS BIBLIOGRÁFICAS

jiensis Morelet) y Sigatoka amarilla (Mycosphaerella musicola Leach) en la cultivariedad de plátano Mbouroukou (África 1). Infomusa 10(2), 3-7.

González, A.M., C. Gómez y M. Aristizábal. 2003. Características de crecimiento y producción de híbridos FHIA en Colombia. Infomusa 12(1), 46-49.

Grisales, F. 2001. África 1: Nueva variedad de plátano para la zona cafetera. Avances Técnicos No. 290, Cenicafé, Chinchiná, Colombia.

Herrera, J.W. y M. Aristizábal. 2003. Caracterización del crecimiento y producción de híbridos y cultivariedades de plátano en Colombia. Infomusa 12(2), 22-24

Hoyos, J.E. y J. Castaño. 2007. Evaluación de la resistencia de Mbouroukou (África 1) y FHIA-03 a las sigatokas negra (Mycosphaerella fijiensis Morelet) y amarilla (Mycosphaerella musicola Leach). Agron. 15(2), 67-76.

ICA. 1996. Cultivo del plátano. Investigación básica. Resultados. Instituto Colombiano Agropecuario, Armenia, Colombia.

Nowakunda, N., P.R. Rubaihayo, M.A. Ameny y W. Tushemereirwe. 2000 Aceptabilidad por parte de consumidores en Uganda, de bananos introducidos. Infomusa 9(2), 22-25.

Ovalle, J.A. y S.G. Rueda. 1999. Manejo post-cosecha del banano criollo (Musa AAA). Serie de paquetes de capacitación sobre manejo post-cosecha de frutas y hortalizas No. 2. Segunda edición. Convenio SENA-Reino Unido, Centro Agroindustrial del SENA, Armenia, Colombia.

Rodríguez, M., J. Morales y J. Chavarriaga. 1985. Producción de plátano (Musa AAB, ABB). Centro Agronómico de Investigación y Enseñanza (CATIE), Turrialba, Costa Rica.

Sierra, L.E. 1993. El cultivo del banano: producción y comercio. Augura, Medellín, Colombia.

Soto, M. 1985. Banano cultivo y comercialización. Litografía e Imprenta Lil, San José, Costa Rica.

Turner, D.W. 1994. Bananas and plantains. pp. 37-64. En: Schaffer, B. y P. Andersen. (eds.). Handbook of environmental physiology of fruit crops. Volume II: Sub-tropical and tropical crops. CRC Press, Boca Raton, FL.

Uthaiah, B.C., M. Indiresh, R. Jayarama y K. Balakrisma. 1992. Performance of banana cultivars under Indian west coast conditions. Agric. Res. J. Kerala 30(2), 84-88. 